\title{
Методологические особенности проведения микросоциальных исследований в цифровую эпоху
}

\author{
О.А. Игнатьева \\ Санкт-Петербургский государственный университет \\ olga7919@mail.ru
}

\section{Аннотация}

Данная статья посвящена особенностям проведения социальных исследований в цифровую эпоху. В статье особенно подчеркивается неравенство возможностей институциональных и индивидуальных исследователей в процессе формирования выборок, отвечающих требованиям репрезентативности и нормальности распределения. В связи с этим на первое место для индивидуальных исследователей и небольших исследовательских коллективов выходят методы непараметрической статистики, позволяющей осуществлять работу с малой выборкой. Исследования, проводимые на такой выборке, носят название микросоциальных исследований. Также в статье особенно подчеркивается возможность использования современного программного обеспечения для обработки данных путем статистического и сетевого анализа. В данной работе рассматриваются возможности применения методов качественного анализа данных, которые приобрели особую популярность в первой четверти XXI века. Речь идет о методах конденсации смысла, дискурс-анализе, методе «обоснованной теории». Автор особенно подчеркивает необходимость формирования исследовательских компетенций, которые позволят корректно проводить как качественные, так и количественные исследования, еще при обучении в высшем учебном заведении. В связи с этим в статье приводится ряд методических рекомендаций по созданию учебных курсов по статистике и методологии исследований, которые могут быть полезны преподавателям и аналитикам.

Ключевые слова: микросоциальные исследования, непараметрическая статистика, сетевой анализ, обоснованная теория, дискурс-анализ, конденсация смысла, Pajek

Библиографическая ссылка: Игнатьева О.А. Методологические особенности проведения микросоциальных исследований в цифровую эпоху // Информационное общество: образование, наука, культура и технологии будущего. Выпуск 4 (Труды XXIII Международной объединенной научной конференции «Интернет и современное общество», IMS-2020 (сборник научных статей). - СПб: Университет ИТМО, 2020. С. 09-21. DOI: 10.17586/2587-8557-2020-4-09-21

\section{Введение}

В деятельности практического исследователя наиболее часто встречаются исследования, проводимые на малой выборке. Такие исследования необходимо обозначить как микроисследования, тем самым проведя грань с широкомасштабными социологическими исследованиями, проводимыми на большой выборке. Для того, чтобы провести полноценное социологическое исследование общественного мнения необходимы ресурсы, которыми обладают только специализированные институционализированные организации такие, как институт Гэллапа в США, институт демоскопии в Германии [1] или ВЦИОМ и Левада-центр в России. Однако большинство независимых исследователей не обладают такими ресурсами и вынуждены работать с малыми выборками. 
Это относится и к студентам высших учебных заведений, которые проводят самостоятельные исследования в рамках написания выпускных квалификационных работ.

Ввиду распространенности микросоциальных исследований, т.е. исследований на малой выборки, необходимо дополнительно организовать процесс обучения с такими типами данных. Подобно разделению социологических исследований на качественные и количественные [2, с. 26], микроисследования также могут быть классифицированы подобным образом. Работа с малой выборкой требует особой подготовки исследователя для работы с таким типом данных. При обработке количественной информации нужно использовать методы непараметрической статистики, о которых речь пойдет далее. Анализ и интерпретация качественных данных, полученных с использованием качественных методов исследования, также требует особых знаний и навыков в использовании специализированных методов. Особенности использования данных методов будут также представлены в данной работе.

\section{1. Особенности формирования выборки в социальных исследованиях}

Любое исследование начинается с четкого формулирования проблемы и гипотезы, которая должна быть адресована ко всей генеральной совокупности [3, с. 37]. При этом научно обоснованный результат можно получить только при анализе выборочной совокупности, которая должна отвечать требованию репрезентативности, т.е. аналогичным образом повторять данные генеральной совокупности.

Проведение социальных исследований предполагает возможность использования разных видов выборок [4, с. 59], среди которых наиболее распространёнными являются случайная выборка, систематическая, целевая и сплошная. В случае случайной выборки подмножество индивидов (выборка) выбирается из большего набора (генеральная совокупность). Каждый индивидуум выбирается случайным образом, так что он имеет одинаковую вероятность быть выбранным на любом этапе в процессе отбора проб. Наиболее распространенной формой систематической выборки является метод равных вероятностей. При таком подходе продвижение по списку обрабатывается циклически, с возвратом к началу после прохождения до конца списка. Выборка начинается со случайного выбора элемента из списка, а затем выбирается каждый $\mathrm{k}$-й элемент, где $\mathrm{k}$ интервал выборки: $\mathrm{k}=\mathrm{N} / \mathrm{n}$ (N-размер генеральной совокупности, $\mathrm{n}$ - размер выборки). Отбор элементов в целевую выборку зависит от цели и задач исследования, а также от решения исследователя. Необходимо отметить, что в случае микросоциальных исследований используется целевая выборка, которая также может быть представлена разными подвидами, о которых будет рассказано далее. В случае сплошной выборки принимается решение исследовать всю совокупность, которая имеет определенный набор характеристик. В таких случаях часто выбирается вся совокупность, которая отвечает определенным критериям и размер которой невелик.

При проведении микроисследований может быть использована как сплошная выборка, так и целевая. Например, при исследовании социальных проблем приемных семей, находящихся на обслуживании в Центре социальной помощи семье и детям следует использовать сплошную выборку ввиду малочисленности данной клиентской группы.

Важными навыками является умения формирования целевой выборки, которая также широко распространена в исследованиях на малой выборке [5, p. 210]. Здесь можно выделить разные подвиды целевой выборки, такие как выборка с максимальной вариацией, гомогенная выборка, выборка типической случая, выборка экстремальных (отклоняющихся) случаев, выборка критического случая, экспертная выборка.

Выборка с максимальной вариацией используется для охвата разных мнений, которые интересуют нас в исследовании, начиная от тех условий (люди, случаи / организации, события), которые считаются типичными, до тех, которые более экстремальны по своей 
природе. Основной принцип, лежащий в основе выборки с максимальными вариациями, заключается в том, чтобы лучше понять феномен, рассматривая его со всех сторон.

Отбор однородных проб направлен на формирование гомогенной выборки; то есть выборки, чьи единицы (например, люди, случаи) имеют одинаковые характеристики (профессия, происхождение, пол). Данный тип выборки используется, когда мы изучаем конкретную группу, конкретные случаи.

Нормальность, типичность изучаемых случаев интересует нас при использовании выборки типического случая (например, людей, сайтов, индивидов, событий). Когда мы используем выборку типического случая - это не значит, что она является репрезентативной как вероятностная выборка, но она может быть полезной при изучении других подобных выборок.

Выборка экстремальных случаев используется для того, чтобы сосредоточиться на случаях, которые являются особыми или необычными, обычно в том смысле, что в этих случаях выделяются заметные результаты, неудачи или успехи. Эти крайние случаи полезны, потому что они часто дают существенную информацию о конкретном явлении, которое может служить уроком (или примером передовой практики), который направляет будущие исследования и практику.

Выборка критического случая особенно полезна в качественных исследованиях, исследованиях с ограниченными ресурсами, а также в исследованиях, где единственный случай может быть решающим при объяснении интересующего явления. Хотя такие критические случаи не следует использовать для статистических обобщений, можно утверждать, что они могут помочь в тщательном создании логических обобщений.

Экспертная выборка применяется, когда данные по выборке собираются от людей с особыми знаниями, т.е. экспертов в своей области. Эти знания могут потребоваться на определенном этапе проведения качественного исследования.

Необходимо заметить, что применение статистических методов не гарантирует получение научных результатов. Поэтому применение традиционной статистики, которая связана с нормальным распределением и требует от исследователя существенных усилий и ресурсов, оказывается для многих исследователей слишком трудным и неблагодарным делом. Непараметрическая же статистика напротив является простой и дешевой в использовании. Её можно применять на выборке менее 10 человек, и она требует относительно простых статистических расчётов.

В случае проведения качественных исследований выборка формируется несколько иначе, что придает ей некоторую субъективность [5, p. 206]. Данная выборка носит направленный характер и называется выборкой по типу снежного кома. Здесь доступные объекты изучения набирают будущие объекты из числа своих знакомых. Таким образом, выборка, кажется, растет как снежный ком. По мере увеличения выборки собирается достаточно данных, которые могут быть полезны для исследования. Этот метод выборки часто используется для работы с группами, к которым исследователям трудно получить доступ, например, к представителям политической или бизнес элиты. Каждого респондента просят порекомендовать вас следующему человеку, который соответствует определенным критериям, например, иметь максимум информации по конкретному аспекту изучаемой проблемы.

\section{2. Количественные методы обработки данных}

Статистический анализ представляет собой совокупность количественных методов обработки информации. Для проведения статистического анализа принято выделять 4 типа данных: номинальные, порядковые, количественные и дихотомические. Номинальные переменные - это категориальные переменные, которые содержат категории, которые нельзя ранжировать. Например, релаксация, улучшение фигуры, снижение веса. 
Порядковые переменные - это переменные, категории которых можно ранжировать, но расстояние между категориями в этом ряду не одинаково или не может быть выражено числами. Например, 1) каждый день, 4-6 дней в неделю; 2) всегда, часто, редко, никогда. Количественные переменные - это метрические переменные, в которых расстояние между категориями идентичны на протяжении всего ряда категорий. Например, возраст, вес, количество минут, проведенное на тренажере. Наконец, дихотомические переменные - это переменные, которые имеют два значения. Например, пол (М, Ж). Поскольку они содержат один интервал, то их можно трактовать, как ординальные (порядковые) переменные, однако хотя чаще всего данные переменные считаются номинальными.

Выделяют три типа статистического анализа: одномерный, двумерный и многомерный. Одномерный анализ - это анализ одной переменной за один раз. К нему относится частотный анализ, меры центральной величины (среднее арифметическое, медиана, мода), меры изменчивости (дисперсия, стандартное отклонение). Двумерный анализ - это анализ двух переменных одновременно, чтобы обнаружить возможную взаимосвязь между ними, также это поиск доказательств того, что вариации в одной переменной связаны с вариациями в другой переменной. К двумерному анализу относится анализ таблиц сопряженности, корреляция и некоторые другие методы вычисления взаимосвязи между двумя переменными. Многомерный анализ соответственно представляет собой анализ взаимосвязи между тремя и более переменными. Его примерами служит многомерный дисперсионный анализ, многомерный регрессионный анализ и многофакторный анализ.

В зависимости от типа данных может быть использован тот или иной вид статистического анализа. При проведении статистического анализа необходимо формулировать нулевую и альтернативную гипотезы, на проверки которых и направлены соответствующие манипуляции с данными.

Статистический анализ используется для обработки результатов анкетирования. После получения сырых данных необходимо закодировать варианты ответов по каждому вопросу. Если вопросы носили открытый характер и получилось много вариантов ответов, их необходимо укрупнить, категоризировать. Например, математическое и инженерное образование могут быть включены в укрупненную категорию «техническое образование». Анкеты в основном предполагают использование номинальных данных (словесных обозначений), которые нельзя ранжировать с точки зрения мало или много, плохо или хорошо. Также в распоряжении исследователя обычно бывают небольшие выборки, что затрудняет использование статистических методов, предназначенных для данных с нормальным распределением.

Развитие непараметрической статистики открывает для исследователей огромные и уникальные возможности [6, с. 342-369]. Она позволяет получить точный результат, отвечающий высоким критериям научности, при использовании малой выборки. При работе с непараметрической статистикой исследователи имеют возможность использовать потенциал SPSS, Statistica, R и других подобных статистических пакетов. Это направление не связано с нормальным распределением и для получения валидных результатов необходимо очень грамотно использовать статистические процедуры.

Для образовательных учреждений преподавание непараметрической статистики должно заключаться в практическом обучении студентов нескольким базовым методам. Здесь могут оказаться полезны: непараметрический критерий сравнения двух выборок UМанна-Уитни, непараметрический критерий множественного сравнения Н-КраскелаУоллеса, коэффициент ранговой корреляции Спирмена, коэффициент ранговой корреляции Кендалла, тест Вилкоксона и тест МакНемара [7].

Для использования непараметрической статистики следует тщательного готовить специалистов, как в рамках основных образовательных программ, так и на курсах дополнительного образования. Для выбора конкретного метода статистического анализа и правильной интерпретации полученных результатов необходимо четкое понимание различий выборок с точки зрения нормальности распределения. 
Для проверки нормальности распределения используются данные асимметрии, эксцесса, которые должны быть не более единицы, либо критерий Колмогорова-Смирнова, либо критерий Шапиро-Уилкса.

Математические модели в структуре научного исследования могут быть подразделены на три типа: измерительные математические модели, описательные математические модели и модели статистического вывода [11, с. 14]. При этом измерительные модели могут быть классифицированы в зависимости от типа шкал: номинальные, порядковые и количественные. Каждому типу данных соответствует своя группа аналитических методов, наибольшее разнообразие которых приходится на данные, измеренные в метрической (количественной) шкале.

Важно отметить, что в случае социологических исследований чаще всего используются номинальные данные, что резко сокращает возможность использования статистических методов. Данном случае для анализа данных применимы таблицы сопряженности, хиквадрат, коэффициент сопряженности, коэффициенты Крамера, анализ частот и соответствия.

В случае количественных данных разнообразие типов описательных математических моделей и коэффициентов гораздо шире. Среди них можно упомянуть и анализ частот, и корреляционный анализ, и сравнительный анализ. Также приобретает смысл использования многомерных моделей. В случае небольшой выборки или нарушения требования нормальности распределения к метрическим данным могут быть применены непарамтерические критерии анализа, которые были перечислены выше.

При этом критерий U-Манна-Уитни и Н-Краскела-Уоллеса используется для сравнения выборок: первый в случае сравнения двух выборок, второй в случае сравнения выборок количеством больше двух.

Критерий непараметрической статистики, используемый в качестве альтернативы tкритерия для сравнения независимых выборок, носит название критерия Манна-Уитни. При использовании данного критерия отсутствуют условия нормального распределения и одинаковости дисперсий, свойственных для параметрической статистики. Критерия Манна-Уитни применим для порядковых данных, что позволяет их ранжировать. Интерпретация результатов похожа на с интерпретацию результатов, получаемых с использованием t-критерия для независимых выборок. Но критерий Манна-Уитни рассчитывается путем попарного сравнения элементов первой выборки с элементами второй. Критерий Манна-Уитни является прекрасной заменой t-критерий Стьюдента в параметрической стаистике.

Критерий Краскела-Уоллиса представляет собой обобщение критерия Манна-Уитни в случае $\mathrm{k}$ несвязанных выборок и используется для оценки отличий между несколькими выборками по уровню определенного признака. Критерий Краскела-Уоллиса представляет собой альтернативу критерию Фишера (F-критерия) в однофакторном дисперсионном анализе для непараметрической статистики. Данный критерий обладает для малой выборки той же чувствительносьтю, что и F-критерий для выборки с нормальным распределением.

Коэффициенты корреляции Спирмена и тау-Кендалла позволяют выявить совместную изменчивость данных, распределение в которых не отвечает нормальному. Преимуществом коэффициента тау-Кендалла является то, что совместную изменчивость признаков можно определить с точки зрения вероятности их появления, а не доли дисперсии [8, с. 78].

Коэффициент ранговой корреляции Спирмена является методом непараметрической статистики, используемым для изучения связи между феноменами. При этом определяется наличие параллельной связи между двумя рядами данных и делается оценка тесноты установленной связи с учетом коэффициента значимости. Если упорядоченные по степени возрастания или убывания показатели совпадают (большему значению одного индикатора соответствует большее значение другого индикатора), исследователь должен сделать 
вывод о наличии прямой корреляционной связи. $\mathrm{B}$ случае противоположной направленности рангов, когда большему значению одного показателя соответствует меньшее значение другого, следует говорить о наличии обратно пропорциональной связи.

Коэффициент ранговой корреляции тау-Кендалла представляет собой метод, альтернативный коэффициенту ранговой корреляции Спирмена. Данный метод используется для определения взаимосвязи между ранговыми переменными. При интерпретации результатов, полученных с использование тау-Кендалла, учитывается разность вероятности совпадения и инверсии в рангах. Значения коэффициента ранговой корреляции для одних и тех же данных обычно слегка больше значения коэффициента Спирмена, чем у тау-Кендалла. При этом уровень значимости обоих коэффициентов будет либо одинаковым, либо несколько отличаться в сторону увеличения у тау-Кендалла.

Критерии МакНемара и Т-Вилкоксона используются для сравнения двух зависимых выборок, которые не отвечают требованиям нормального распределения. При этом критерий МакНемара действует для таблицы 2х2. Критерий МакНемара представляет собой метод, схожий с параметрическим критерием t-Стьюдента и с непараметрическим критерием Т-Вилкоксона. Он используется для анализа связанных измерений. При этом в отличии от критерия хи-квадрат, критерий МакНемара применяется только тогда, когда условие о независимости выборок не выполняется, когда признак учитывается выполняется на одних и тех же объектах.

Критерий Т-Вилкоксона для связанных выборок (критерий суммы рангов Вилкоксона) является непараметрическим статистическим критерием, который используется для сравнения двух связанных выборок по уровню определенного признака, измеренного либо в непрерывной, либо в порядковой шкале. Метод предполагает сопоставление абсолютных величин выраженности сдвигов в прямом или противоположном направлении. Для того, чтобы использовать данный критерий, в начале необходимо проранжировать абсолютные величины сдвигов, а затем суммировать ранги. Когда сдвиги являются случайными, суммы их рангов будут примерно одинаковы. При наличии закономерностей величина сдвигов в одну сторону будет выше, чем в обратное, что не характерно для случайных сдвигов.

Современным методом исследования в социальных и политических науках является метод сетевого анализа [9]. Он позволяет визуализировать отношения между акторами. При его использовании также можно работать с небольшой выборкой. В отличие от статистического анализа исследования с использованиями данного метода относятся к разведывательному типу исследования и не предполагают создание гипотезы с самого начала. Несмотря на сходство с качественной методологией в смысле возможности интерпретации, основанной на визуализации данный метод можно отнести к методам количественного исследования, так как используемое для него программное обеспечение Pajek, Ucinet позволяют рассчитывать соответствующие показатели (центральности, централизацию, плотность сетей и т.д.) [10]. Несмотря на всю инновационность метода, его использование носит трудоемких характер. Во-первых, это сложность сбора данных об акторах, будущих узлах сети. Во-вторых, это интерпретация данных, полученных в результате сетевого анализа. В первом случае мы используем методы ивент и контентанализа для выявления акторов и построения взаимосвязей между ними. Метод интервью становится для нас недоступным в связи с социальной дистанцией статусных акторов. Для интерпретации результатов необходимо использовать теоретические модели исследователей, которые пытаются решить проблему объяснительного потенциала сетевого анализа.

Таким образом, микросоциальные исследования представляют собой случаи с малой выборкой, для обработки результатов которой требуется использование методов непараметрической статистики, рассмотренных в данном разделе. Во всех случаях использования данных методов пороговое значение ошибки, разделяющей статистически 
достоверные различия от статистически недостоверных, лучше устанавливать на уровне 0,05, чтобы избежать статистической ошибки второго рода.

\section{3. Методы качественной обработки данных}

Качественными исследованиями являются такие исследования, в ходе которых собранный материал представлен словесным, номинальными данными. Обычно качественные исследования проводятся на малой выборке ввиду трудоемкости сбора и обработки такого материала. Для формирования выборки в качественном исследовании используется метод снежного кома, когда каждый последующий респондент попадает в выборку на основании рекомендаций предыдущего. Для проведения качественного исследования используются методы глубинного интервью, включенного наблюдения, фокус-группы, экспертного опроса и кейс-стади. Метод глубинного интервью является более трудозатратным, чем проведение фокус-группы, однако позволяет добраться до нюансов жизненного мира информанта, так как отсутствует эффект групповой динамики $[11$, c. 121]. Однако в случае проведения фокус-группы опытным модератором, существует возможность позитивно использовать данный эффект, собрав гораздо большее количество информации по данной теме за тот же промежуток времени.

Для анализа и интерпретации качественных данных исследователи могут прибегать к феноменологическому и герменевтическому подходу. Но наиболее эффективными являются следующие методики качественного анализа данных: конденсация смысла, категоризация значений, нарративное структурирование, обоснованная теория и дискусранализ. Именно эти методики лучше использовать в микроисследовании.

Конденсация смысла является самой простой методикой выражения смысла сказанного автором, как в письменной, так и устной форме. «Как уже отмечали, техника конденсации смысла наряду с техникой кодировки и категоризации относится к «сквозным» техникам, которые применяются в большинстве качественных подходов» [12, с. 224]. Она будет особенно полезна начинающим исследователям. Метод конденсации смыслов используется для выделения основного смысла в естественных смысловых единицах. Данными единицами могут являться или абзацы текста, или тематические разделы транскрипта интервью. После выделения конденсированного смысла можно определить основные темы данного материала, выстроить логическую цепочку изложения материала, не пропустив основных идей автора и не сделав акцент на вторичные аспекты изложенного текста.

Категоризация значений предполагает выделение категорий в полученном нарративе. Эта методика хорошо подходит для исследований, сочетающих применение качественных и количественных методов. Категоризация является методом отнесения познаваемого объекта к некоторому классу (категории). При этом категории выражают наиболее существенные закономерности в определенных областях действительности. Категоризация охватывает как материальные объекты, так и чувственные образы, стереотипы мышления и поведения.

Актуальным является рассмотрение проблемы категоризации с точки зрения культурно-исторического процесса, ее понимания как основной составляющей познания и восприятия, осуществляемых в контексте смыслополагающей и коммуникационной деятельности. В этом отношении важно учитывать опыт социальных наук, открывающий многообразные формы упорядочивания знания на всех уровнях человеческого бытия и мышления.

Понятие «категоризация» получило широкое распространение в различных видах человеческой деятельности и опыта, характеризуя мыслительные способности человека в форме классифицирования, упорядочивания воспринимаемой и познаваемой действительности, возможности предвидения возникновения объектов реального и воображаемого мира. 
Существуют разные способы категоризации: психологические, логические и математические. Но остается нерешенной главная проблема: какие критерии стоит использовать при осуществлении категоризации. Решение данной проблемы не ограничивается классификацией на основе понятий, но изменяется в зависимости от специфики изучаемых областей.

Нарративное структурирование является перестройка всего нарратива, т.е. создания из него нового текста, в котором последовательно излагается смысл сказанного [13, с 86]. Разрозненные и хаотично распределенные по тексту интервью смысловые части сказанного информантом превращаются единый, последовательный рассказ. Интерпретация значений является наиболее ненадежной методикой качественного анализа, поскольку допускает вольные толкования сказанного. Однако именно она обладает наибольшим позитивным эвристическим потенциалом. Она позволяет извлекать из нарратива глубинных интервью очень неочевидные смыслы, которые имеют для науки огромную ценность. И такие результаты можно получать на малой выборке.

Метод «обоснованной теории» используется для обработки данных, полученных методом кейс-стади [14, с. 143-160]. Это способ создания эмпирических теорий, который предполагает упорядочивание данных путем их прочтения посредствам сплошного, осевого и выборочного кодирования. Сплошное кодирование: делается первичное прочтение и на полях ставятся заметки (мемосы - короткие комментарии, чувства, идеи, слова). Использование осевого кодирования дает возможность соединить эти слова поновому, устанавливая взаимоотношения между категориями и субкатегориями, которые имеют причинно-следственный характер. Этап выборочного кодирования является завершаеющим и предполагает собой разработку центральной категории. Выявленная центральная категория будет направлять дальнейший сбор данных и ее соотнесение только с теми кодами, которые имеют с ней связь.

После кодирования всех необходимых данных предполагается развитие и насыщение центральной категории: выделение свойств категории и ее измерения. Каждая субкатегория имеет характеристики (свойства и измерения). При этом используется несколько основных категорий и множество соподчиненных им субкатегорий. Например, если субкатегорией является «Регистрация малого предприятия», то ее свойствами становятся стоимость бизнеса в денежном выражении, сложность оформления представляемой документации, продолжительность юридической процедуры, необходимость действовать незаконными методами. При этом измерение означает характеристику стоимости в денежном выражении, т.е. цифры в рублях.

Далее идет построение концепций посредством интерпретаций выделенных категорий. Это создание блоков, из которых выстраивается здание «Обоснованной теории». На этом этапе следует переходить к рассмотрению причин и следствий. В процессе интерпретации происходит наделение смыслом, делаются выводы (исходя из здравого смысла, официальных мнений и теорий), предлагаются объяснения, включая конкурирующие точки зрения.

И наконец, проводится верификация концепции в той среде, на изучение которой она направлена, а также в научном сообществе. Полученная теория выносится на обсуждение. Вначале идет внешняя верификация на принятие, т.е. теория выносится на суд тех, кто будет ее использовать в практической деятельности. Далее осуществляется «внутренняя верификация», т.е. сопоставление своих результатов с имеющимися подобным разработками в научной литературе.

И наконец, еще одним методом анализа транскрипта интервью или научного текста является дискурс-анализ. В науке известны следующие основные подходы к дискусранализу: дискусрсивная теория Э. Лакло и Ш. Муфф, критический дискурс-анализа, дискурсивная психология и ТРУД (транскрипция устного дискурса) М.Л. Макарова. В дискурс в лингвистике означает связанные по смыслу два или несколько предложений. По мнению Л. Филлипса и М. Йоргенсена, «дискурс означает организацию языка, 
в соответствии со структурами, свойственными высказываниям людей в различных сферах социальной жизни» [15, с. 25].

Дискурс-анализ - это собой критическое изучение дискурса для выявления его смысла в соответствии с использованными там структурами. Другими словами, дискуср-анализ это анализ языка в его использовании, т.е. в социокультурном контексте. Предпосылками дискуср-анализа являются наши знания и язык. Результатом исторических взаимоотношений людей являются наши знания о мире. Дискурс представляет собой форму социального поведения, которая служит для репрезентации социального мира. Разное социальное поведение - это результат разного понимания мира. Например, наводнение - это факт материального мира. Но как только люди пытаются определить значение этого события, оно попадает в рамки дискурса.

Кто-то описывает это явление в терминах метереологического дискурса, связав поднятие уровня воды с ливнем, кто-то в терминах политического дискусра, связав наводнение с плохим управлением, кто-то в религиозном дискурсе, характеризуя наводнение как признак апокалипсиса. Придание того или иного значения в дискурсе создает наш мир или изменяет его.

Механизмом, создающим и изменяющим мир, служит язык. В структурной лингвистике Фердинанда де Соссюра знаки имеют две грани, выраженные в форме (означающее) и в содержание (означаемое). Отношение между формой и содержанием носит произвольный характер, оно является результатом договоренности между людьми. Так собака - это всего лишь обозначение лающего животного принятого в обществе. При этом форма знака может меняться в зависимости от языка, содержание тоже. Например, разное обозначение собаки в зависимости от языка и применение термина собака к человеку. Соссюр выделял два уровня языка: Langue (язык) - это фиксированная и неизменная структура как основа лингвистики, Parole - это ситуативная речь.

Исследователи из Западной Европы ориентированы на изучение дискурса как текста, исследователи из США на изучение дискурса как речи. Российские исследователи анализирует дискурс как речь и текст. Поструктуралисты считают, что именно в обыденной речи есть те структуры, которые необходимо изучать.

Основными задачами дискурс-анализа является изучение устных и письменных форм дискурсов в естественных условиях общения, зафиксированных в текстах или транскриптах, а также исследование предметно-содержательной стороны языковой коммуникации в социокультурном контексте.

В дискурс-анализе существуют проблемы сбора информации, транскрипции дискурса и парадокс наблюдателя. Для решения проблемы сбора необходимого и достаточного количества достоверной информации проводят репрезентативную выборку и триангуляцию. Триангуляция - это сравнение результатов анализа данной выборки, полученных посредством дискурс-анализа с результатами анализа, полученными другими методами. Проблема транскрипции выражается в том, что в процессе транскрибирования человек может ненамеренно исказить дискурс через призму своего восприятия. На Западе существуют базы данных, содержащих как транскрипты интервью, так и дискурсы. Например, ВCET (Birmingham Collection of English text). Парадокс наблюдателя означает, что, когда люди знают, что за ними наблюдают, их речь становится менее непринужденной. В процессе транскрипции ученые могут фиксировать непривычные формы общения и оставлять без внимания привычные.

При анализе транскрипта интервью методом дискурс-анализа по системе ТРУД М. Макарова [16], принято выделять следующие единицы дискурс-анализа: акт; ход; обмен; трансакция; речевое событие. Итак, речевой акт представляет собой единицу социокультурного поведения в процессе взаимодействия, основными характеристиками которой являются намеренность, целеустремленность и конвенциональность. Коммуникатиный ход - это вербальнное/невербальное действие одного из участников, продвигающее общение к достижению цели. 
Макроречевой акт - это комплекс речевых актов. Дискурсивный акт - это минимальная коммуникативная единица (речевая/жесто-мимическая), которая влияет на развитие речи. Обмен - это двухстороння единица анализа (вопрос-ответ, просьба-обещание, приветствие-приветствие). Трансакция - это единица анализа, превышающая по объему сложные обмены. Событие - это единица макроуровня дискурса. Все вышеуказанные единицы анализа относятся к социально-интерактивным единицам.

Таким образом, в существующей практике проведения качественных исследований сложился набор наиболее востребованных и оправданных методов обработки полученных данных, среди которых наибольшее распространение получили метод конденсации смысла, категоризации значения, нарративного структурирования, метод обоснованной теории и дискурс-анализа. Данные методы предполагают не только систематизацию, но и интерпретацию данных. В основном эти методы позволяют корректно обработать транскрипты интервью, в то время как метод конденсации смыслов и дискурс-анализ также подходят для анализа научных текстов. Метод обоснованной теории также используется для анализа данных, полученных в ходе включенного наблюдения, для которого характерно ведение дневника и возможность использования свободного интервью для прояснения значения и контекста увиденного. При этом некоторые исследователи полагают, что последнюю лучше делать с точки зрения первичной интерпретации (т.е. объяснения с позиций самого информанта). Это приведет к объективной реконструкции жизненного мира исследуемого и будет соответствовать принципу феноменологической редукции.

\section{Заключение}

Формирование научно-исследовательских навыков необходимо начинать еще в высшем учебном заведении. Для этого необходимо уделить особое внимание построению курсов по исследовательским дисциплинам. В нашей работе мы предлагаем некоторые советы, которые стоит учесть преподавателям в своей методической работе над исследовательскими дисциплинами. Создание рабочей программы дисциплины обычно основано на требованиях к развитию компетенций соответствующего профессионального стандарта. Формирование навыков проведения количественных и качественных исследований, а также способы осуществления интерпретации данных обязательно входят в стандарт специалистов социального профиля и могут быть развиты как в рамках основных образовательных программ, так и при реализации программ повышения квалификации и профессиональной переподготовки в виде дополнительного образования.

Итак, обратимся к составлению курсов статистическому анализу. Отдельным важным компонентом обучения студентов социологическим микроисследованиям является обучение использованию программного обеспечения (SPSS, STATISTIKA, R). Для целей овладения навыком проведения микроисследований обучение статистическим пакетам необходимо проводить обучение нескольким видам статистического анализа. Остальные вида анализа студенты смогут впоследствии освоить па аналогии.

При построении курса по статистическом анализу слушатели и студенты, в первую очередь, должны познакомиться со шкалами данных и четко определять их в данных полевых исследований. Также необходимо четко определить такие виды статистических исследований как одномерное, двухмерное и многомерное. Часть курса должна быть посвящена особенностям обработки результатов, полученных в ходе микросоциальных исследований. Как уже было сказано выше, данный тип исследования связан с использование сравнительно небольшой выборки, но при этом довольно часто встречается в социальных науках. Несмотря на нарушения требования нормальности распределения, требование репрезентативности выборки является обязательным. Такая выборка должна повторять процентное соотношение единиц, входящих в генеральную совокупность. Кроме описания типов выборок и способов их формирования при микроисследованиях, 
особое внимание необходимо уделить таким методам непараметрической статистики как коэффициент ранговой корреляции Пирсона, тау-Кендалла, критериям Краскела-Уоллеса, Манна-Уитни, МакНемара и Вилкоксана. При работе с номинальными данными часть времени должна быть отведена преподаванию таблиц сопряженности и выявлению значимых отношений на основе критериев Хи-квадрата, Фи и Ви Крамера. При работе с малой выборкой коэффициент значимости лучше выставлять на уровне 0,05 , так как получить значимые связи на уровне 0,01 является весьма проблематичным.

При составлении плана по дисциплине «Методология исследования» особое внимание следует уделить качественным методам сбора и обработки информации. Качественное исследование, по мнению российского социолога В.И. Ильина [17], сродни искусству, поэтому его методология сопоставляется с драматургией. Для того, чтобы получить надежные данные необходимо научиться грамотно составлять дизайн исследования, выделяя в нем объект, предмет, цели и задачи. Важно правильно сформулировать гипотезу исследования, так как она влияет на качественный состав выборки. Также важно определить способы формирования теоретической выборки, одной из разновидностью которой является выборка по методу снежного кома. Необходимо отметить, что в ходе проведения качественного исследования, обычно получаются номинальные (качественные) данные. Конечно, их можно анализировать общетеоретическими методами анализа и синтеза, однако более правильно уделить внимание качественным методам обработки данных, получивших широкое распространение в последнее время. Желательно включить в курс метод конденсации смыслов, нарративное структурирование, категоризацию значений, обоснованную теорию и дискурс-анализ. Все эти методы идеально подходят для анализа транскриптов глубинного и свободного интервью. При этом метод обоснованной теории может быть использован для анализа кейсов и включенного наблюдения. Помимо упорядочивания данных, он позволяет создавать мини-теории эмпирического уровня. Метод конденсации смыслов подходит как для анализа транскриптов, так и для анализа научных документов.

Современный мир диктует свои условия, и на авансцену выходят новые методы, для которых цифровое пространство является необходимым условием их использования. Речь идет о сетевом анализе. Сегодня при составлении курсов по методологии нельзя обойти стороной данный метод. Сети становятся основой взаимодействия в современном мире как в социальной, так и политической сфере. Сетевой анализ дает возможность не только визуализировать данные, но и рассчитать количественные взаимосвязи между теми или иными акторами, найти брокеров сети, вычислить престиж. Метод является инновационным, но требует обращения к интерпретационным моделям М. Минтрома, Дж. Баумгартнера, П. Сабатье и Л. Карлссона для усиления своего объяснительного потенциала, который является его слабым местом.

Также в процессе обучения необходимо уделить внимание развитию навыков обучающихся по предоставлению результатов исследования в виде научных статей и презентаций. Результаты, представленные в данных формах, должны исчерпывающе отражать проделанную работу, учитывать основные достижения и не быть перегруженными излишней информацией. Для представления результатов исследования в виде презентаций необходимо освоить Microsoft Power Point, а в некоторых случаях и Adobe Acrobat. Навыки написания научных статей следует тренировать включением в качестве заданий к самостоятельно работе подготовку рецензий.

\section{Литература}

[1] Ноэль Э. Массовые опросы: введение в методику демоскопии. М.: Прогресс, 1978. $153 \mathrm{c}$.

[2] Добреньков В.И., Кравченко А.И. Методы социологического исследования: учебник. М.: Инфра-М, 2016. 72 с. 
[3] Ядов В.А. Стратегия социологического исследования: описание, объяснение, понимание социальной реальности: учеб.пособие. М.: Издательство «Омега-Л», 2009. $567 \mathrm{c}$.

[4] Сергеева И.И. Статистика / И.И.Сергеева, Т.А. Чекулина, С.А. Тимофеева. М.: ИнфраM, 2016. $272 \mathrm{c}$.

[5] Babbie E. The Basics of Social Research. Wadsworth: Cengage Learning, 2014. 542 c.

[6] Брайман А., Белл Э. Методы социальных исследований. Группы, организации, бизнес / Пер. с англ. Харьков: Изд-во Гуманитарный центр, 2012. 762 с.

[7] Ермолаев О.Ю. Математическая статистика для психологов: учебник. М.: Московский психолого-социальный институт: Флинта, 2003. 336 с.

[8] Наследов А.Д. Математические методы психологических исследований: анализ и интерпретация данных: учеб. пособие / А.Д. Наследов. СПб: Речь, 2004. 392 с.

[9] Сморгунов Л.В. Политические сети: теория и методы анализа: учебник для студентов высших учебных заведений, обучающихся по направлению подготовки ВПО 030200 "Политология" / Л. В. Сморгунов, А. С. Шерстобитов. М.: Аспект Пресс, 2014. 318 с.

[10]De Nooy W., Mrvar A., Batagelj V. Exploratory Social Network Analysis with Pajek: Revised and Expanded Edition for Updated Software. Third Edition. Cambridge: Cambridge University Press, 2018. 509 p.

[11]Квале С. Исследовательское интервью. М.: Смысл, 2003. 301 с.

[12] Бусыгина Н.П. Методология качественных исследований в психологии: учеб. пособие. М.: Инфра-М, 2013. 304 с.

[13]Бусыгина Н.П. Качественные и количественные методы исследования в психологии: учебник для бакалавриата и магистратуры. М.: Издательство Юрайт, 2018. 423 с.

[14]Масалков И.К., Семина М.В. Стратегия кейс стади: методология исследования и преподавания. М.: Академический Пропект; Альма Матер, 2011. 443 с.

[15]Йоргенсен М.В. Дискурс-анализ. Теория и метод: пер. с англ. Харьков: Изд-во "Гуманитарный центр", 2008. 352 с.

[16]Макаров М. Л. Основы теории дискурса. М.: ИТДГК «Гнозис», 2003. 280 с.

[17]Ильин В.И. Драматургия качественного полевого исследования. СПб.: Интерсоцис, 2006. $256 \mathrm{c}$.

\title{
Methodological features of microsocial research in the digital age
}

\author{
O.A. Ignatjeva
}

\section{Saint Petersburg State University}

This article is about the features of conducting social research in the digital age. The article especially emphasizes the inequality of opportunities for institutional and individual researchers in the process of generating samples that meet the requirements of representativeness and normality of distribution. In this regard, the methods of nonparametric statistics that allow working with a small sample come out in the first place for individual researchers and small research teams. Studies conducted on such a sample are called microsocial studies. The article also emphasizes the possibility of using modern software for data processing through statistical and network analysis. This paper discusses the possibility of using methods of qualitative data analysis, which gained particular popularity in the first quarter of the XXI century. We are talking about the methods of condensation of meaning, discourse analysis, the method of "grounded theory". The author especially emphasizes the need to form research competencies that will allow for the conduct of both qualitative and quantitative research correctly, while still studying at a higher educational institution. In this regard, the article provides a number of guidelines for the creation of training courses on statistics and research methodology, which may be useful to teachers and analysts. 
Keywords: microsocial studies, nonparametric statistics, network analysis, grounded theory, discourse analysis, sense condensation, Pajek

Reference for citation: Ignatjeva O.A. Methodological features of microsocial research in the digital age // Information Society: Education, Science, Culture and Technologies of the Future. Vol. 4 (Proceedings of the XXII International JointScientific Conference «Internet and Modern Society», IMS-2020, St. Petersburg, June 17-20, 2020). - St. Petersburg: ITMO University, 2020. P. 09 - 21. DOI: 10.17586/2587-8557-2020-4-09-21

\section{Reference}

[1] Noel E`. Massovy`e oprosy`: vvedenie v metodiku demoskopii [Mass surveys: introduction to demoscopy methods]. Moscow: Progress, 1978. (in Russian).

[2] Dobren kov V.I., Kravchenko A.I. Metody' sociologicheskogo issledovaniya: uchebnik [Sociological research methods: textbook]. Moscow: Infra-M, 2016. (in Russian).

[3] Yadov V.A. Strategiya sociologicheskogo issledovaniya: opisanie, ob" "yasnenie, ponimanie social'noj real'nosti: ucheb.posobie [Sociological research strategy: description, explanation, understanding of social reality: manual]. Moscow: Publishing House "Omega-L", 2009. (in Russian).

[4] Sergeeva I.I., Chekulina T.A., Timofeeva S.A. Statistika [Statistics] / I.I.Sergeeva, T.A. Chekulina, S.A. Timofeeva. Moscow: Infra-M, 2016. (in Russian).

[5] Babbie E. The Basics of Social Research. Wadsworth: Cengage Learning, 2008.

[6] Brajman A., Bell E`. Metody` social’ny`x issledovanij. Gruppy`, organizacii, biznes [Methods of social research. Groups, organizations, businesses] / Per. s angl. Kharkov: Publishing House "Humanitarian Centre", 2012. (in Russian).

[7] Ermolaev O.Yu. Matematicheskaya statistika dlya psixologov: uchebnik [Mathematical statistics for psychologists: textbook]. Moscow: Publishing House of Moscow Psychological Institute: Flinta, 2003. (in Russian).

[8] Nasledov A.D. Matematicheskie metody` psixologicheskix issledovanij: analiz i interpretaciya danny'x: ucheb. posobie [Mathematical Methods of Psychological Research: Data Analysis and Interpretation:a manual] / A.D. Nasledov. Saint Petersburg: Rech [Speech], 2004. (in Russian).

[9] Smorgunov L.V., Sherstobitov A.S. Politicheskie seti: teoriya i metody`analiza: Uchebnik dlya studentov [Political Networks: Theory and Analysis Methods: A Textbook for Students] / L.V. Smorgunov, A.S. Sherstobitov. Moscow: Publishing House «Aspekt Press», 2014. (in Russian).

[10] De Nooy W., Mrvar A., Batagelj V. Exploratory Social Network Analysis with Pajek: Revised and Expanded Edition for Updated Software. Third Edition. Cambridge: Cambridge University Press, 2018.

[11] Kvale S. Issledovatel`skoe interv`yu [Research Interview]. Moscow.: Smy`sl [Sense], 2003.

[12] Busy`gina N.P. Metodologiya kachestvenny`x issledovanij v psixologii: ucheb. posobie [Methodology of qualitative research in psychology: a textbook.]. Moscow: Infra-M, 2014.

[13] Busy`gina N.P. Kachestvenny`e i kolichestvenny`e metody` issledovaniya v psixologii: uchebnik dlya bakalavriata i magistratury' [Qualitative and quantitative research methods in psychology: textbook for undergraduate and graduate studies]. Moscow: Publishing House "Uright", 2017. (in Russian).

[14] Masalkov I.K., Semina M.V. Strategiya kejs stadi: metodologiya issledovaniya i prepodavaniya [Case-stage strategy: research and teaching methodology]. Moscow: Akademicheskij Propekt; Al’ma Mater, 2011. (in Russian).

[15] Jorgensen M.V. Diskurs-analiz. Teoriya i metod [Discourse analysis. Theory and method]: per. s angl. Kharkov: Publishing House "Humanitarian Centre", 2008. (in Russian).

[16] Makarov M. L. Osnovy' teorii diskursa [Foundation of discourse theory]. Moscow: ITDGK «Gnozis», 2003. (in Russian).

[17] Il' in V.I. Dramaturgiya kachestvennogo polevogo issledovaniya [Quality field research dramaturgy]. Saint Petersburg.: Intersocis, 2006. (in Russian). 